\title{
An End-to-End QoS Adaptation Architecture for the Integrated IntServ and DiffServ Networks
}

\author{
Ing-Chau Chang ${ }^{1}$ and Shi-Feng Chen ${ }^{2}$ \\ ${ }^{1}$ Department of Computer Science and Information Engineering, National Changhua \\ University of Education, Changhua, Taiwan, R.O.C. \\ icchang@cc.ncue.edu.tw \\ ${ }^{2}$ Institute of Information Management, Chaoyang University of Technology, Wufeng, \\ Taichung County, Taiwan, R.O.C.
}

\begin{abstract}
In this paper, we propose an end-to-end quality of service (QoS) adaptation network architecture to guarantee service qualities for mobile users, according to user requirements and available network resources on the end-toend path which is across the wired backbone DiffServ network and wireless IntServ networks. Further, we adopt the bandwidth broker (BB) for resource allocations and COPS-SLS protocol for negotiating QoS in DiffServ backbone, and the context transfer protocol (CTP) on IntServ wireless networks to resolve service interruptions during handoffs. Finally, we propose a flexible and efficient bandwidth adjustment algorithm, which is based on CBQ and RED schemes, to adaptively reallocate available bandwidth among different traffic classes on border gateway routers between DiffServ and IntServ networks.
\end{abstract}

\section{Introduction}

It has been shown as a challenging task to provide end-to-end QoS services for users on the traditional wired network environment which is composed of access networks with the IntServ scheme [1] and the backbone network with the DiffServ [2] one [3]. Problems such as incompatibilities of these two different QoS schemes may result in breakdowns of QoS mechanisms for packets of multimedia streams in the boundary between DiffServ and IntServ domains. Researches proposed mechanisms to resolve these problems by designing an integrated framework to complement these two QoS schemes [3-4], e.g., the traffic mapping mechanism in edge routers which connect IntServ and DiffServ domains. As wide-spreading wireless networks, which adopt the IntServ QoS scheme, have become major access networks for mobile hosts $(\mathrm{MH})$ in recent years, the aforementioned problems will get even worse due to the mobility of MH. Whenever the MH, who adopts the mobile IP (MIP) protocol for mobility management, hands over to a new wireless cell, the $\mathrm{MH}$ will suffer handoff latencies to acquire a new care-of address (CoA) and then execute binding update (BU) to its home agent (HA) before resuming the media stream through the new path. Further, the end-to-end path between the $\mathrm{MH}$ and the correspondent node $(\mathrm{CN})$ may change, which then introduces extra operations to guarantee the end-to-end QoS on this new path. If network resources on the new path are not available, the real-time multimedia service for the $\mathrm{MH}$ will be interrupted, which may degrade its QoS significantly. 
For guaranteeing the end-to-end QoS of the moving $\mathrm{MH}$ on the integrated IntServ wireless and DiffServ wired network environment, we summarize the following four necessary criteria:

1. There must be QoS signaling protocols between different IntServ and DiffServ network domains.

2. Because IntServ and DiffServ domains adopt totally different approaches to process user packets, there must be traffic mapping mechanisms between two domains to provide packets with the same QoS.

3. For reducing the handoff latency when the $\mathrm{MH}$ hands over to a new cell, there must be seamless handoff mechanisms to avoid media playback interruption.

4. There must be QoS and service adaptation mechanisms to dynamically adjust endto-end service qualities of the $\mathrm{MH}$ to available network resources when the handoff occurs. If redundant resources are allocated to other class users, networks should re-adjust resources among all classes of MHs to satisfy the requested QoS of the high-priority $\mathrm{MH}$ as much as possible.

This paper is organized as follows. In section 2, we will give a survey for works on the end-to-end QoS and compare their pros and cons. In section 3, we will propose and discuss detail operations of our end-to-end QoS adaptation framework to fulfill the above four criteria. Simulation results will be shown in section 4 to exhibit efficiencies of this framework. We conclude this paper in section 5.

\section{Related Works}

IETF IntServ usually combines RSVP to negotiate and reserve resources on network routers by keeping the soft state information of each flow. It can achieve true per-flow QoS guarantees on small networks like LANs. However, because each IntServ router has to record the soft state information for each flow, it is not an efficient approach to deploy IntServ on WAN. Moreover, as the number of active flow changes, IntServ is not flexible enough due to the re-negotiation process of RSVP. For resolving IntServ's scalability problem, IETF proposed DiffServ for achieving per-class QoS by handling most of QoS operations on edge routers. As soon as user packets enter the DiffServ domain, the edge router in the domain boundary will classify these packets into different traffic classes by marking them with corresponding DiffServ code points (DSCP), according to the service level agreement (SLA) made when the user subscribes services with the network ISP. Depending on DCSP values in packet headers, the interior core router schedules these packets with different precedence values, which are called as their per-hop-behaviors (PHB). Consequently, DiffServ is more appropriate on wide-area backbone networks than on local-area networks.

For integrating the wired DiffServ backbone and IntServ LANs to achieve end-toend QoS support, researches [3-4] work on the traffic mapping issue by proposing the common open policy service (COPS) [5] as the signaling protocol to dynamically negotiate user requirements and resource allocations with the bandwidth broker (BB) [6] on the DiffServ domain. RSVP control messages on the IntServ domain are not 
processed in the DiffServ one to reduce complexities on core routers. However, without considering how to manage user mobility on wide-spreading wireless LANs which adopt the IntServ approach, these works only satisfy criteria 1 and 2.

Trossen and Chaskar [7] proposed to use Session Initial Protocol (SIP) [8] for initial session negotiation between the $\mathrm{MH}$ and the transmitter under heterogeneous networks. While the MH is going to change its network attachment point, the current access router (AR) of the mobile will directly transfer the user information to the new AR. With this kind of application context transfer, this approach reduces the original SIP latency for re-negotiation. Consequently, it satisfies criteria 1 and 3. However, this work has not mentioned how to handle the inconsistent situation when the new AR cannot support the same amount of resources as the current one does, which fails to meet criterion 4. Further, the authors omitted mechanisms of how to map traffics on different domains with their SIP-based approach, which violates criterion 2.

The network architecture proposed by Chaouchi and Pujolle [9] also consists of a DiffServ backbone network and IntServ wireless LANs. If MHs want to enable new services or change service requirements, they will adapt MCOPS (Mobile COPS) as a signaling protocol, which meets criterion 1 . As for criterion 2 , they defined a direct traffic class mapping between the WLAN and the DiffServ. Major contributions of this paper are Anticipated Handoff and Adaptive Handoff for criteria 3 and 4, respectively. The anticipated handoff uses user requirements recorded in the policy base of the server to predict possible moving directions and work on the handoff proactively when the mobile entered the overlapped area of two wireless cells. Depending on resources of the mobile's new location, the adaptive handoff adjusted QoS of the MH at the new location or reserved resources for the user in advance.

\section{System Architecture}

Characteristics of our proposed architecture, shown in Fig. 1, are listed as follows:

(1) The wired backbone is based on IETF RFC 2998 DiffServ architecture and each DiffServ domain is equipped with a bandwidth broker (BB) [6] to manage internal resources and search for the optimal route in the domain.

(2) Local wireless networks are configured to support IntServ. We use RSVP as an end-to-end signaling protocol because RSVP is a receiver-oriented one, which is appropriate to handle the change of resource requirements after the MH's handoff.

(3) For supporting uninterrupted services after the MH's handoff, we integrate the Context Transfer Protocol (CTP) [10], which was proposed by SEAMOBY [11], to transfer the MH's current context from the old access router (AR) to the new one for accelerating the handoff process as soon as CTP receives the layer 2 handoff trigger. Further, we modify the original context by adding new QoS parameters such that the new AR is able to optimally allocate its network resources to meet requirements of this moving mobile for continuing its services. In this way, our architecture fulfills criterion 3 .

(4) We adapt COPS-SLS [12], which inherits concepts of COPS, as a negotiation protocol to dynamically adjust resources between wired and wireless networks. With it, criterion 1 can be achieved. 


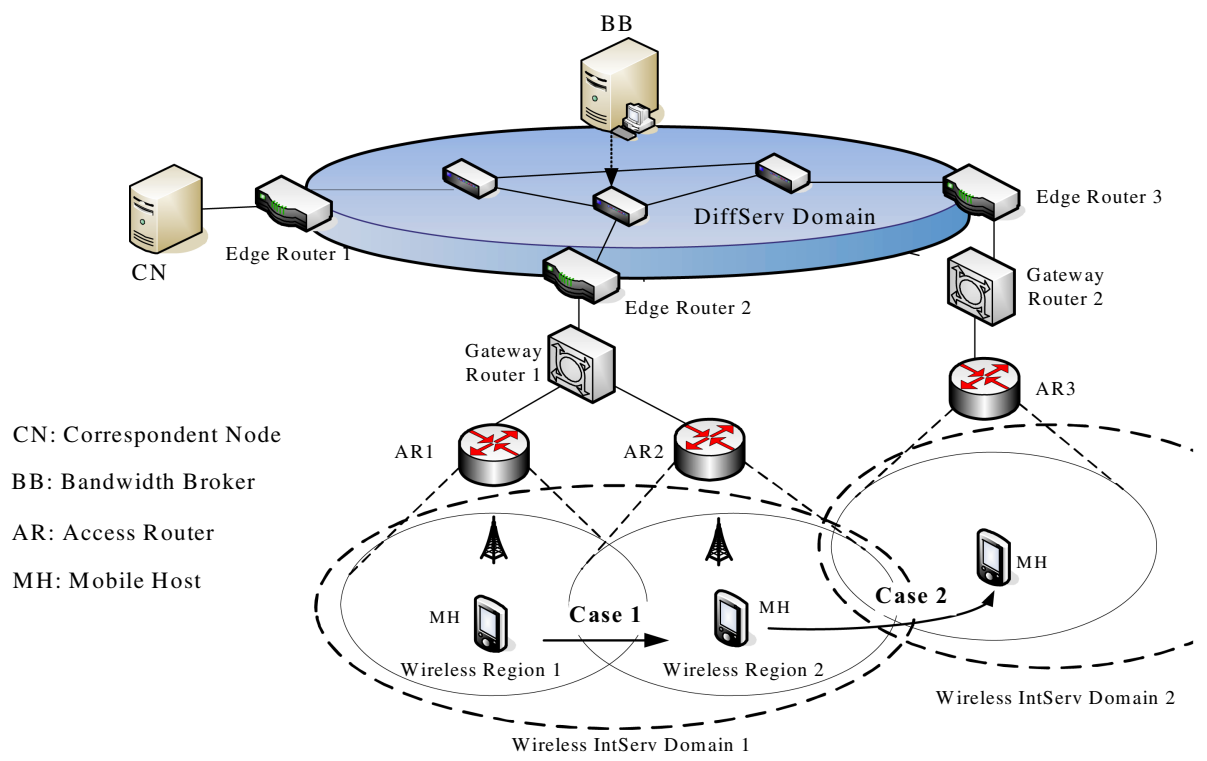

Fig. 1. The proposed end-to-end QoS adaptation architecture

\subsection{Proposed Handoff Flow}

In this proposed architecture, the following procedures shown at the first stage for RSVP initialization in Fig. 2 must be executed to start a service:

a. When the MH wants to start its service, the CN will use RSVP to issue a PATH message at step 1.1 in Fig. 2, which contains TSPEC, PHOP (Previous Hop Router Address) [13] and ADSPEC for QoS, to the MH. This PATH message is considered as a normal traffic one when it passes through the DiffServ domain.

b. After the MH receives the RSVP PATH message from the $\mathrm{CN}$, it will send back an RSVP RESV message along the reverse path of PATH. While this message arrives at the edge router, i.e., ER2, of the DiffServ domain, it will retrieve QoS requirements from this message and convey them to the BB by COPS-SLS at step 1.2. The BB will record these QoS requirements and corresponding policy configurations. After that, the edge router will forward the RESV message back to the $\mathrm{CN}$ to complete the end-to-end signaling process at step 1.3.

c. $\mathrm{CN}$ begins its transmission to the $\mathrm{MH}$ at step 1.4.

In the following, we will describe how to achieve the end-to-end QoS adaptation in our proposed architecture for the Intra-IntServ-domain handoff and Inter-IntServdomain handoff.

Case 1. As shown in Fig. 2, when the MH moves from the service range of AR1 to AR2 under the same Gateway Router1, this kind of handoff is called Intra-IntServdomain handoff with the following five processing stages. 


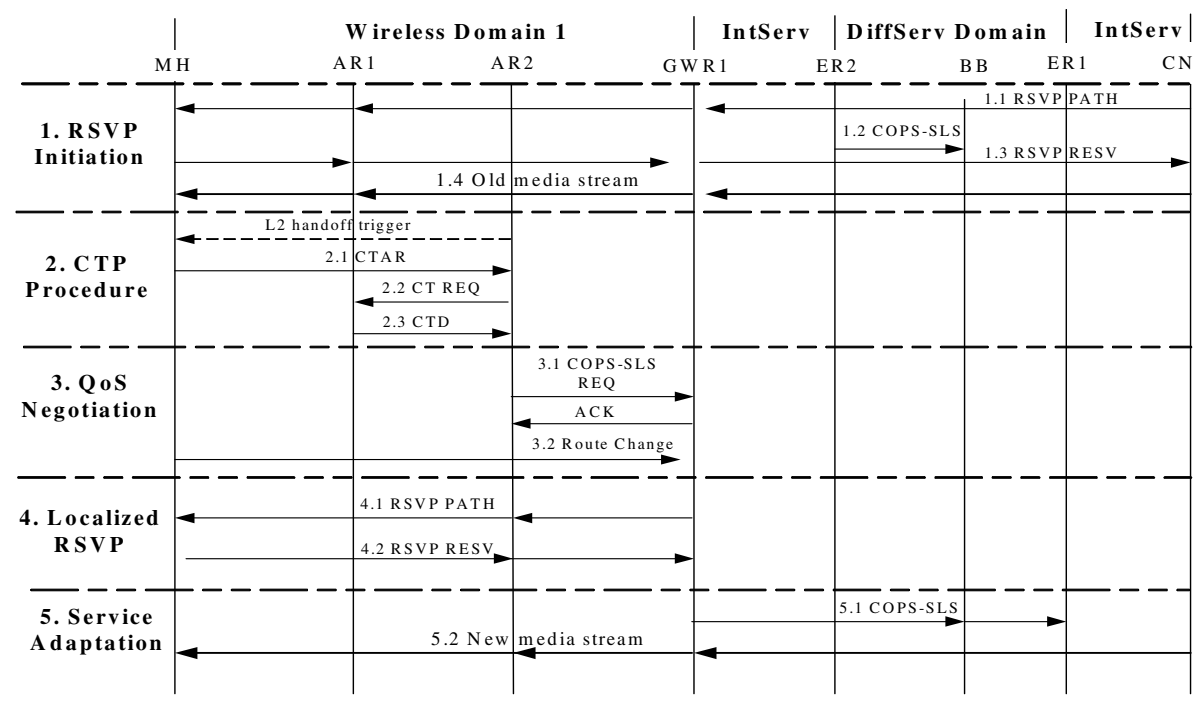

Fig. 2. Flow of the Intra-IntServ-domain handoff

(1) RSVP initialization

As mentioned above, RSVP procedures must be executed to start a service.

(2) Handoff Start and CTP Procedure

a. When the MH enters the overlapped range between the wireless region 1 and 2 under the wireless IntServ domain 1, it recognizes it is going to perform the Intra-IntServ-domain handoff process within the IntServ domain 1.

b. While the $\mathrm{MH}$ receives the beacon signal, i.e., Layer 2 handoff trigger, of AR2 in the wireless region 2, the CTP process starts. As shown in Fig. 2, the MH will issue a Context Activate Request (CTAR) to AR2 at step 2.1 and then AR2 will send a Context Request (CT Request) to the original AR1 in the wireless region 1 at step 2.2. At last, AR1 will forward the MH's context in the modified CTD message back to AR2 at step 2.3. The modified CTD message is shown in Table 1, including RSVP QoS parameters like the Service Class, RSPEC and TSPEC.

Table 1. Modified CTD format

\begin{tabular}{|c|c|c|c|c|c|}
\hline Messa & Yype & $\mathrm{C}$ & $\mathrm{R}$ & Rsv & Length \\
\hline \multicolumn{6}{|c|}{ Elapsed Time (in milliseconds) } \\
\hline \multicolumn{6}{|c|}{ Mobile Node's Previous Care-of Address } \\
\hline \multicolumn{6}{|c|}{ Mobile Node's New Care-of Address, if C=1 } \\
\hline $\begin{array}{c}\text { Type }= \\
\text { Auth }\end{array}$ & $\begin{array}{c}\text { Type } \\
\text { Length }\end{array}$ & & & gorithm & $\begin{array}{c}\text { Key } \\
\text { Length }\end{array}$ \\
\hline $\begin{array}{c}\text { Service } \\
\text { Class }\end{array}$ & \multicolumn{2}{|c|}{ RSPEC } & \multicolumn{3}{|c|}{ TSPEC } \\
\hline
\end{tabular}


(3) QoS Negotiation and Handoff Completion

As AR2 receives the MH's context, it will try to provide enough resources to the $\mathrm{MH}$. If AR2 cannot fulfill MH's resource requirements, it will degrade QoS of the MH and notify Gateway Router 1 of the degraded QoS and the changed route to the destination by COPS-SLS for continuing services of the $\mathrm{MH}$ at AR2, which is shown at step 3.1. Oppositely, the MH at AR2 still owns the original QoS as at AR1 if AR2 has enough network resources. Because AR2 and Gateway Router 1 do not reserve resources for the $\mathrm{MH}$ in advance, the $\mathrm{MH}$ has to issue a Route Change message to Gateway Router 1 via AR2 for recording the current location of the $\mathrm{MH}$ to be in the wireless region 2, as shown at step 3.2.

(4) Localized RSVP Procedure

a. As Gateway Router 1 receives the Route Change message from the $\mathrm{MH}$, it has recognized that the $\mathrm{MH}$ has moving to the new wireless region 2.

b. For reconstructing the local path and reserving resources in the new wireless region, Gateway Router 1 will convey a new RSVP PATH message to the MH under AR2 at step 4.1 and the MH will return the RESV message back to Gateway Router 1 after accepting the PATH message at step 4.2.

(5) Service Adaptation

If the localized RSVP procedure changes network resources allocated to this $\mathrm{MH}$, GWR1 will adopt COPS-SLS to transmit modified QoS requirements to BB in the DiffServ backbone domain. Then BB will update configurations in the MH's policy database and issue modified policies to ER1 by COPS-SLS. Then, ER1 will adjust priorities, i.e., DSCP values, of $\mathrm{MH}$ packets entered into the DiffServ domain according to these modified policies at step 5.1. Finally, the media stream will follow the new path to the MH via GWR1 and AR2 as shown at step 5.2. In this way, our architecture fulfills criterion 4.

Case 2. When the $\mathrm{MH}$ moves from the service range of AR2 under Gateway Router 1 of the IntServ domain 1 to AR3 under Gateway Router 2 of the IntServ domain 2, which is called Inter-IntServ-domain handoff, our architecture will perform the following five processing stages as shown in Fig. 3.

(1) RSVP initialization

As mentioned above, RSVP procedures must be executed to start a service.

(2) Handoff Start and CTP Procedure

This stage is similar to stage 2 of the Intra-IntServ-domain handoff, except operations are executed between the original AR2 in IntServ domain 1 and the new AR3 in the IntServ domain 2 as shown in Fig. 3. Steps to send CTAR, CT Request and CTD messages are numbered as step 2.1, 2.2 and 2.3 respectively.

(3) QoS Re-negotiation and Handoff Completion

a. As AR3 receives the MH's context and it cannot fulfill MH's resource requirements, it will degrade the MH's QoS and notify Gateway Router 2 in the new IntServ domain of the degraded QoS by COPS-SLS for continuing services of the $\mathrm{MH}$ at AR3, which is shown at step 3.1 in Fig. 3.

b. Gateway Router 2 will allocate the most appropriate resources after negotiation between the MH's requirements and its current available resources. Then it will adopt COPS-SLS to transmit negotiated QoS parameters to the BB in the DiffServ domain, as shown at step 3.2 in Fig. 3. 
c. Oppositely, if AR3 can fulfill resource requirements of the $\mathrm{MH}$, the $\mathrm{MH}$ has to issue a Route Change message to Gateway Router 2 via AR3 in the IntServ domain 2 as shown at step 3.3.

d. As Gateway Router 2 receives the Route Change message from AR3, it will recognize the $\mathrm{MH}$ is performing an Inter-IntServ-domain handoff such that it will further issue a Route Change message to the $\mathrm{CN}$ for re-executing the end-to-end RSVP signaling at step 3.3.

(4) Re-routing and Re-configuration

a. Whenever the $\mathrm{BB}$ in the DiffServ domain receives negotiated QoS parameters from Gateway Router 3, it adopts SNMP/OSPF to re-configure resources among interior routers and find a new optimal route for the $\mathrm{MH}$ to satisfy its QoS, i.e., QoS routing, in the DiffServ domain, which is shown at step 4.1.

b. Then BB will update the MH's policy database and issue modified policies to ER1 by COPS-SLS. Finally, ER1 will adjust DSCP values of MH packets entered into the DiffServ domain as shown at step 4.2.

(5) End-to-End RSVP Signaling

a. As soon as the $\mathrm{CN}$ receives the Route Change message from Gateway Router 2 and recognizes the $\mathrm{MH}$ has moving to a new IntServ domain, it has to release old resources and re-execute the new end-to-end RSVP signaling process.

b. The CN will issue a new PATH through Gateway Router 2 and AR3 to the MH and the $\mathrm{MH}$ will return an RESV back to the $\mathrm{CN}$ as shown at steps 5.1 and 5.2 respectively. In this way, this architecture provides the media stream an end-toend QoS adaptation in the integrated networks as shown at step 5.3.

\subsection{Traffic Mapping Mechanism}

In the integrated IntServ and DiffServ networks, traffics of user services will pass through IntServ for the wireless LAN and DiffServ for the wired backbone. In IntServ, three different traffic types are defined as the guaranteed service (GS), control load service (CLS) and best effort (BE). DiffServ also provides expedited forwarding (EF), assure forwarding (AF) and best effort (BE) services for classifying user packets and determining priorities of them, according to the multi-fields classifier which consist of characteristics of these packets or payment policies chosen by users.

For reducing the processing overhead to convert the traffic type from the IntServ domain to the DiffServ domain, and vice versa, at the edge router, our architecture follows the traditional concept to provide an one-to-one traffic mapping in Table 2 between these two domains. Because both the GS traffic in IntServ and the EF one in DiffServ aim to provide the steady and seamless service, we map these two traffics directly. For the CLS traffic in IntServ, we further propose three sub-classes, i.e., CLS Real-time $\left(\mathrm{CLS}_{\mathrm{RT}}\right)$, CLS Multimedia $\left(\mathrm{CLS}_{\mathrm{MM}}\right)$ and CLS Control Message $\left(\mathrm{CLS}_{\mathrm{CM}}\right)$, and map them into three different AF classes, i.e., AF1 to AF3, with different queue management priorities in DiffServ. According to the work done by AQUILA [14], we also define corresponding rules for traffic parameters and algorithms for our traffic mapping. In this way, our architecture fulfills criterion 2. 


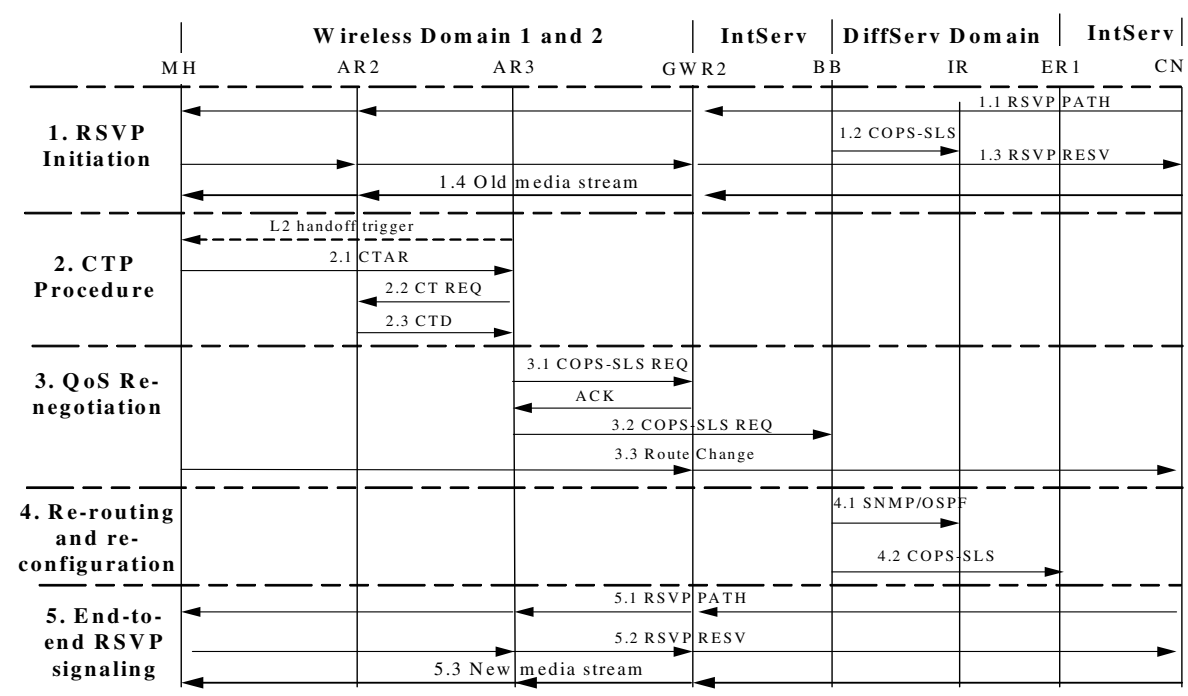

Fig. 3. Flow of the Inter-IntServ-domain handoff

Table 2. Traffic mapping table in our approach

\begin{tabular}{|c|c|c|}
\hline \multicolumn{2}{|c|}{ Traffic Class } & Service Descriptions \\
\hline IntServ & DiffServ & IntServ/DiffServ \\
\hline $\mathrm{GS}$ & $\mathrm{EF}$ & Virtual Leased Line \\
\hline CLS $_{\mathrm{RT}}$ & \multirow{2}{*}{$\mathrm{AF}_{1 \sim 3}$} & Real-time Traffic \\
\cline { 1 - 1 } CLS $_{\mathrm{MM}}$ & & Multimedia Data \\
\cline { 1 - 1 } CLS $_{\mathrm{CM}}$ & & Control Message \\
\hline $\mathrm{BE}$ & $\mathrm{BE}$ & General Packet \\
\hline
\end{tabular}

\subsection{QoS and Service Adaptation}

For providing dynamic QoS and service adaptation, we adopt the class-based queuing (CBQ) [15] and random early detection (RED) [16] to manage traffic queues in the gateway router which manages underlying wireless LANs. In CBQ, a single queue is facilitated for each traffic class, which may contain multiple user connections with similar characteristics. Each class can borrow its available network bandwidth to another class if allowed in its initial configuration. On the other hand, RED provides the queue management mechanism for forthcoming congestion. It will first mark or drop packets when they arrive at the queue and may notify the traffic source to reduce the emission rate for mitigating the congestion. By integrating the multi-queue management of RED and bandwidth sharing of CBQ, we propose an adaptation algorithm, which is denoted as CBQ+RED in this paper, in the IntServ gateway router to handle the situation when the associated queue of the user traffic does not have enough bandwidth to meet the user's requirement. This algorithm is shown in Table 3. 
Table 3. The proposed CBQ+RED QoS adaptation algorithm

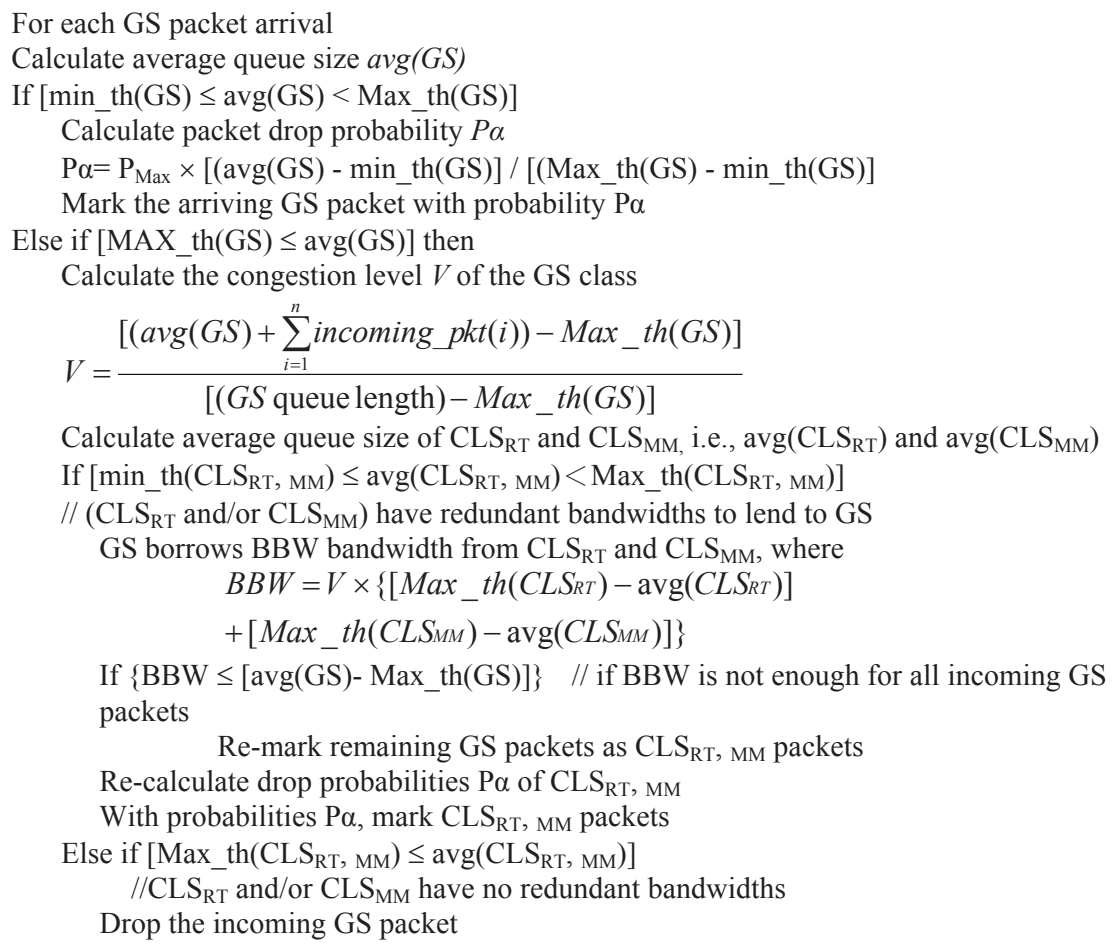

In the following, we will take the GS traffic as an example to explain how this algorithm works. Packets of the GS traffic have the highest priority among all traffic classes in IntServ. As the sum of the average queue length $\operatorname{Avg}(G S)$ and the incoming packet length of GS traffic is approaching to its maximal threshold Max_th(GS) in RED, the dropping probability of the incoming packet is raised accordingly. At last, any incoming GS packet will be dropped when the GS queue length is equal to Max_th(GS). Hence, we modify the CBQ bandwidth sharing mechanism to solve the dropping problem of GS packets by borrowing sparse bandwidth from lower-priority CLS classes. The amount of borrowed bandwidth is determined by the congestion value (V) of the GS queue in Equation 1. The larger value of V means the congestion in the GS queue is more severe because the sum of the average GS queue length and incoming GS packet lengths is larger than Max_th(GS) such that incoming packets have higher probabilities to be dropped. If it happens, our algorithm will first try to borrow redundant bandwidths from classes with lower priorities.

$$
V=\frac{\left[\left(\operatorname{avg}(G S)+\sum_{i=1}^{n} \text { incoming_pkt }(i)\right)-\operatorname{Max} \_t h(G S)\right]}{\left[(G S \text { queue length })-M a x_{-} \operatorname{th}(G S)\right]}
$$

Where $n$ is the number of incoming GS packets in the past. 
This algorithm has to consider the following two issues:

(1) Which class with the next lower priority has redundant bandwidth to lend?

(2) What amounts of bandwidth can be borrowed from this class?

Because arrival times and amounts of incoming packets are depending on specific traffic characteristics of active services, our algorithm uses the congestion level of GS class queue, i.e., the value of $\mathrm{V}$, to calculate the borrowed bandwidth (BBW) from CLS $_{\mathrm{RT}}$ and CLS $_{\mathrm{MM}}$, as formulated in Equation 2.

$$
B B W=V \times\left\{\left[M a x_{\_} t h\left(C L S_{R T}\right)-\operatorname{avg}\left(C L S_{R T}\right)\right]+\left[\operatorname{Max} \_t h\left(C L S_{M M}\right)-\operatorname{avg}\left(C L S_{M M}\right)\right]\right\}
$$

If the next priority CLS classes do not have enough redundant bandwidth to lend to GS, our algorithm will then degrade remaining high-priority GS packets to be lowpriority CLS ones by re-marking them to continue their services, instead of dropping them immediately in traditional RED. However, if CLS classes are also congested, our algorithm is obligated to drop the incoming GS packets.

\section{Simulation}

As mentioned above, our proposed architecture provides the gateway router in the wireless region with the integrated CBQ+RED QoS adaptation algorithm to dynamically adjust user traffics to an appropriate class queue for the best QoS guarantee and wireless bandwidth utilization. In this section, we will execute simulations to exhibit results of this proposed algorithm.

\subsection{Simulation Environment}

With the MATLAB v7.01 and its embedded Simulink for designing the module of our class queues, we conduct this simulation in the gateway router of the IntServ domain with five queues for the GS, CLS $\mathrm{RT}_{\mathrm{RT}}, \mathrm{CLS}_{\mathrm{MM}}, \mathrm{CLS}_{\mathrm{CM}}$ and $\mathrm{BE}$ traffic classes. We assume the bandwidth of each wireless region is $11 \mathrm{Mbps}$, which is further divided into five parts for these five CBQ class queues, as listed in Table 4. The Max_th value of each class queue is $90 \%$ of its allocated bandwidth. Moreover, different class queues are assumed to handle packets with different packet length distributions. GS packet lengths are ranged from $32 \mathrm{~Kb}$ to $256 \mathrm{~Kb}$ with the average value of $190 \mathrm{~Kb}$; CLS $_{\mathrm{RT}}$ and $\mathrm{CLS}_{\mathrm{MM}}$ packet lengths are ranged from $32 \mathrm{~Kb}$ to $256 \mathrm{~Kb}$ with the average value of $136 \mathrm{~Kb}$. The $\mathrm{CLS}_{\mathrm{CM}}$ class is assumed to have the shortest packet lengths of $32 \mathrm{~Kb}$, which means most small-size control messages can reach its destination without suffering congestion and packet dropping at the gateway router.

Table 4. Bandwidth allocations for five class queues at the gateway router

\begin{tabular}{cccccc}
\hline Queue Class & GS & CLS $_{\mathrm{RT}}$ & CLS $_{\mathrm{MM}}$ & CLS $_{\mathrm{CM}}$ & $\mathrm{BE}$ \\
Proportion & $30 \%$ & $25 \%$ & $20 \%$ & $15 \%$ & $10 \%$ \\
Queue size (KB) & 3,300 & 2,750 & 2,200 & 1,650 & 1,000 \\
\hline
\end{tabular}




\subsection{Simulation Results}

Simulation results for consumed bandwidths of GS and CLS $\mathrm{RT}_{\mathrm{RT}}$ over simulation times without using the integrated CBQ+RED QoS adaptation algorithm in the gateway router are shown in Fig. 4. As described above, Max_th(GS) and Max_th(CLS $\left.S_{\mathrm{RT}}\right)$ are set as $90 \%$ of GS and CLS $_{\mathrm{RT}}$ allocated bandwidths, which are $2970 \mathrm{Kbps}$ and $2475 \mathrm{Kbps}$ and illustrated as two horizontal dotted lines in Fig. 4. As shown at 40 second in Fig. 4, the GS queue length is close to its Max_th limit of 2970Kbps and this class is considered as congested. Most incoming large-size GS packets will be dropped to keep the GS queue length below its Max_th limit. However, because the $\mathrm{CLS}_{\mathrm{RT}}$ queue length is below $1500 \mathrm{~Kb}$, which is much less than its Max_th limit of $2475 \mathrm{Kbps}$ at the same time, it means the CLS $\mathrm{RT}_{\mathrm{RT}}$ class has low bandwidth utilization and owns redundant bandwidth that can lend to the GS class.

Fig. 5 shows simulation results by applying our CBQ+RED scheme on GS and $\mathrm{CLS}_{\mathrm{RT}}$ packets at the gateway router. The congestion of GS traffic occurs at $40 \mathrm{sec}-$ ond in Fig. 4 does not happen again with CBQ+RED. It is because the Max_th limit of the GS traffic is raised from $2970 \mathrm{~Kb}$ to $3425 \mathrm{~Kb}$ by borrowing $70 \%$, i.e., the current value of $\mathrm{V}$, of redundant bandwidth, i.e., $(2475 \mathrm{~Kb}-1824 \mathrm{~Kb}=651) \times 70 \%=455 \mathrm{~Kb}$, from the $\mathrm{CLS}_{\mathrm{RT}}$ traffic. In this way, the GS queue continuously accepts incoming GS packets such that its queue length is increased until the new Max_th limit of the GS traffic is reached at 57 second. Oppositely, though the Max_th limit of the CLS $S_{\mathrm{RT}}$ traffic is decreased from $2475 \mathrm{~Kb}$ to $2020 \mathrm{~Kb}$ after lending its $455 \mathrm{~Kb}$ to the GS traffic, the $\mathrm{CLS}_{\mathrm{RT}}$ class still performs well without suffering congestion. As listed in Table 5, our CBQ RED scheme at 57 second can increase the total bandwidth utilization by $6 \%$ as compared to the traditional scheme without $\mathrm{CBQ}+\mathrm{RED}$.

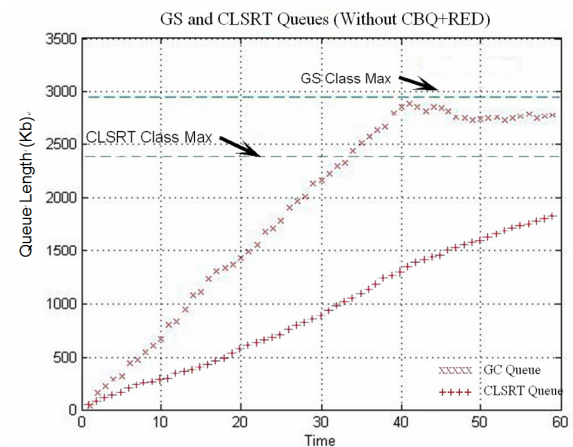

Fig. 4. Queue lengths of the GS and CLS $S_{\mathrm{RT}}$ classes without CBQ+RED

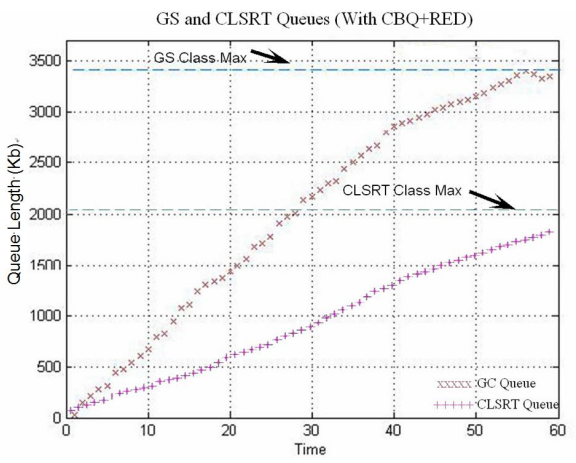

Fig. 5. Queue lengths of the GS and $\mathrm{CLS}_{\mathrm{RT}}$ classes with $\mathrm{CBQ}+\mathrm{RED}$

Table 5. Bandwidth utilization at 57 second with or without the CBQ+RED scheme

\begin{tabular}{cc}
\hline Without CBQ+RED & With CBQ+RED \\
\hline Bandwidth Utilization $(2,765+1,745) / 11,000=41 \%$ & $(3,425+1,745) / 11,000=47 \%$ \\
\hline
\end{tabular}




\section{Conclusions}

In this paper, we propose an end-to-end QoS adaptation architecture with four capabilities: 1. the CTP for fast handoff; 2. the COPS-SLS as the coordination protocol between IntServ and DiffServ; 3. the CBQ+RED scheme to provide better service for the higher-priority traffic class; 4. the receiver-oriented RSVP as the end-to-end signaling protocol to continue service after the user hands over to a new wireless region. This architecture can guarantee the QoS of the higher-priority traffic class and increase total bandwidth utilization for the integrated networks.

\section{References}

1. Braden, R., Clark, D., Shenker, S.: Integrated Services in the Internet Architecture: an Overview. IETF RFC 1633 (1994)

2. Blake, S., Black, D., Carlson, M., Davies, E., Wang, Z., Weiss, W.: An Architecture for Differentiated Services. IETF RFC 2475 (1998)

3. Bernet, Y., Ford, P., Yavatkar, R., Baker, F., Zhang, L., Speer, M., Braden, R., Davie, B., Wroclawski, J., Felstaine, E.: A Framework for Integrated Services Operation over Diffserv Networks. IETF RFC 2998 (2000)

4. Bernet, Y.: The Complementary Roles of RSVP and Differentiated Services in the FullService QoS Network. IEEE Communications Magazine 38(2), 154-162 (2000)

5. Durham, D., Boyle, J., Cohen, R., Herzog, S., Rajan, R., Sastry, A.: The COPS (Common Open Policy Service) Protocol. IETF RFC 2748 (2000)

6. Nichols, K., Zhang, L.: A Two-bit Differentiated Services Architecture for the Internet. RFC 2638 (1999)

7. Trossen, D., Chaskar, H.: Seamless Mobile Applications across Heterogeneous Internet Access. In: IEEE International Conference on Communications, pp. 908-912 (2003)

8. Handley, M., Schulzrinne, H., Rosenberg, J.: SIP: Session Initiation Protocol. IETF RFC 2543 (1999)

9. Chaouchi, H., Pujolle, G.: A New Handover in the Current and Future Wireless Networks. IEICE TRANS. Communication E87-B(9) (2004)

10. Loughney, J., Nakhjiri, M., Perkins, C., Koodli, R.: Context Transfer Protocol (2003), https://www1.ietf.org/internet-drafts/draft-ietf-seamoby-ctp-03.txt

11. Seamoby Group, http://www.ietf.org/html.charters/seamoby-charter.html

12. Liu, C., Liu, Y., Qian, D., Li, M.: An Approach of End-to-End DiffServ/MPLS QoS Context Transfer in HMIPv6 Networks. In: IEEE Eighth International Symposium on Autonomous Decentralized Systems, pp. 245-254 (2007)

13. Moon, B., Aghvami, H.: Reliable RSVP PATH Reservation for Multimedia Communications under an IP Micromobility Scenario. IEEE Wireless Communications 9(5), 93-99 (2002)

14. AQUILA, http://www.ist - aquila.org/

15. Zheng, Q.: A Differentiated Service Supported Bandwidth Allocation Algorithm for Multiple Points Communications. In: IEEE 10th International Conference on Computer Supported Cooperative Work in Design, pp. 1-8 (2006)

16. Chen, W., Li, Y., Yang, S.H.: An Average Queue Weight Parameterization in a Network Supporting TCP Flows with RED. In: 2007 IEEE International Conference on Networking, Sensing and Control, pp. 590-595 (2007) 